\title{
Modelling of the thermal response of a multi-tray food self-heating unit
}

\author{
S. H. Ho ${ }^{1,2}$, M. M. Rahman ${ }^{2}$ \& A. K. Sunol ${ }^{3}$ \\ ${ }^{I}$ Department of Mechanical, Materials and Aerospace Engineering, \\ University of Central Florida, USA \\ ${ }^{2}$ Department of Mechanical Engineering, University of South Florida, \\ USA \\ ${ }^{3}$ Department of Chemical and Biomedical Engineering, \\ University of South Florida, USA
}

\begin{abstract}
This paper presents the modelling and numerical simulation of heat transfer in a multi-tray food self-heating unit for fielded group meals. The unit includes a stack of four tray sets contained in a corrugated cardboard box. Each tray set has a polymeric tray containing a different type of food, a heater inserted beneath it, and a heating tray that houses both of them. The heater generates heat based on a water-activated exothermic chemical reaction. The original heating profile was derived from an approximate analytical solution of the equations of chemical reaction and energy conservation and had the form of an exponential decay function. It was curve fitted against experimental data found from literature for actual values used in simulation. A system of governing equations for transient heat conduction in the composite medium of the stack of four tray sets was solved numerically for temperature distribution within the entire domain. Rectangular- and triangular-shaped heating profiles were also considered for a parametric study on heating profiles. Solutions for different heating profiles were compared. It was found that heat generation of each heater significantly affects the thermal response of a food self-heating unit. The results show that a proper combination of heating generation from different heaters can improve the thermal performance of the unit while reducing cost and weight. The results are useful for designing and optimizing multi-tray food self-heating units.
\end{abstract}

Keywords: food self-heating, exothermic chemical reaction, heat conduction. 


\section{Introduction}

Self-heating food technology has been developed for more than a half of a century. Caldwell and Gillies [1] in 1950 described investigations leading to a satisfactory reaction mixture for heating food compositions incorporated in cans, which could be charged with soups or other liquid foods, and several million of these cans were used during war. In the UK, it was reported in 1960 [2] that selfheating cans originally developed as a wartime expedient was made available to the general public for use wherever other forms of heating were not suitable. Products include three soups and two beverages available to rally drivers, campers, picnickers, etc. Presently, there are many self-heating products that are commercially and widely available, including both food and drink. Oliver-Hoyo et al. [3] presented a classroom activity where a self-heating beverage and the Meals, Ready to Eat (MRE) were used as a real-life chemistry problem. Most of the developments of self-heating food products are presented in the form of patents. Kolb [4] described an insertable thematic module for self-heating cans. This heater contains a liquid reactant and a solid reactant, which is calcium oxide combined with a wax-based inhibitor. Pickard et al. [5] presented a self-heating group meal assembly that can hold a number of food pouches and used exothermic chemical heaters that contained $\mathrm{Mg}-\mathrm{Fe}$ alloy. Lamensdorf [6] proposed a heater using a powder mixture of $\mathrm{Mg}-\mathrm{Fe}$ alloy that improved its performance as demonstrated by the included experimental data.

The Unitized Group Ration-Express ${ }^{\mathrm{TM}}\left(\mathrm{UGR}-\mathrm{E}^{\mathrm{TM}}\right)$ module is a compact, selfheating unit that provides a complete, hot meal for up to 18 soldiers in remote locations $[7,8]$. It has been approved by all departments of the U.S. Department of Defense [7] and available for procurement since 2007 [9]. UGR-E was developed by the U.S. Army based on the Flameless Ration Heater (FRH) technology [10] to heat the food. Detail specifications of the heaters and the food-heating system are described in [11]. A collection of military specifications related to UGR-E is available at [12]. The UGR-E heating unit consists of a stack of four tray sets, put in a corrugated cardboard container box. Each tray set includes a heating tray, made of high-density polyethylene (HDPE), that houses a polymeric food tray and an activation fluid pouch at its bottom. All four activation fluid pouches of the four tray sets are connected to the activator tab. The main component of the UGR-E heating system is the heater, which contains a magnesium-iron compound that can be activated by a saline solution to initiate an exothermic chemical reaction. When food heating is required, four heaters (previously packed separately) are slipped beneath the food trays into the spaces between each pair of heating tray and food tray. Then the cover of the container box is closed. As the activation tab is pulled, activation fluid floods the bottom of the trays and activates all the heaters simultaneously. Once the heaters are activated, the chemical reaction generates heat to get the meals ready in up to 45 minutes.

Most of the study and development in the field of food self-heating employ the experimental approach, which is practical and suitable for fast design to market applications. However, numerical modelling and simulation is a powerful 
tool to improve and optimize the designs of the products. This paper presents a model and simulation results for the problem of a self-heating unit of four food trays for group meals. Figure 1 presents a one-dimensional (1D) model of a UGR-E unit [11]. As shown in fig. 1, the $x$-axis direction is along the height of the stack of the tray sets, from bottom to top. The dimensions (thicknesses) and material properties of the components are given in table 1 . The total height of the domain is $210 \mathrm{~mm}$.

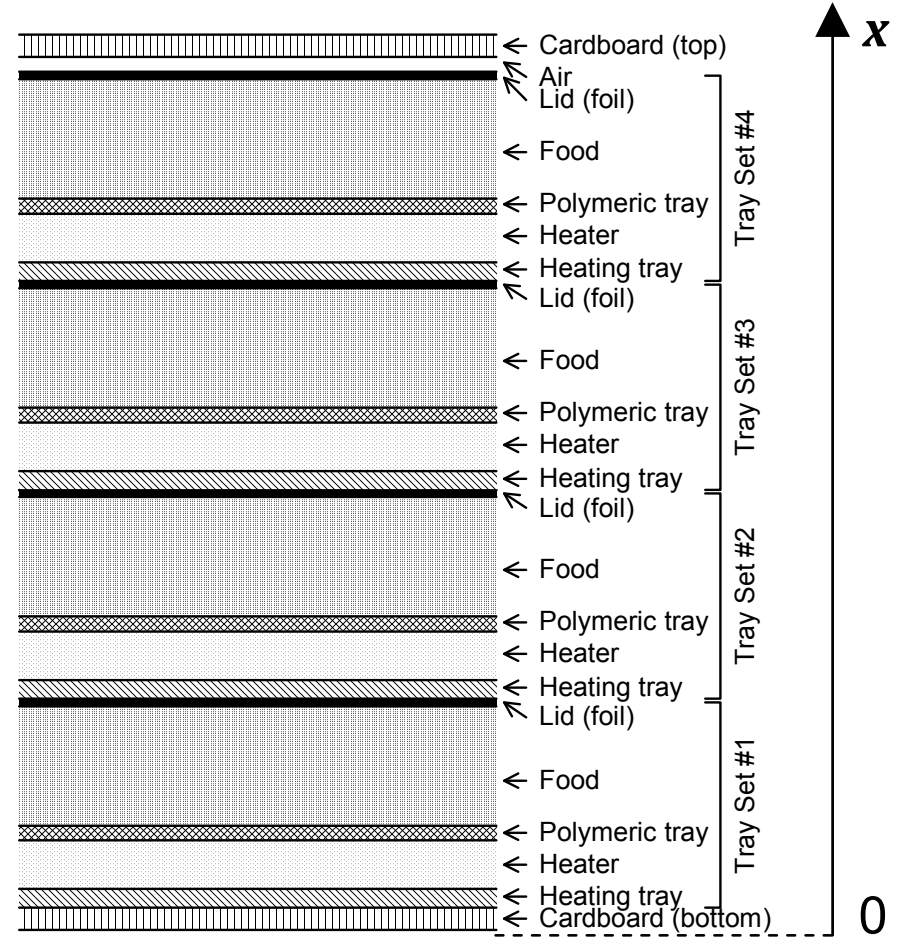

Figure 1: One-dimensional model of food self-heating system for group meals.

Table 1: Thickness and thermophysical properties of components.

\begin{tabular}{|l|c|c|c|c|}
\hline Component (material) & $\begin{array}{c}\text { Thickness } \\
\delta, \mathrm{mm}\end{array}$ & $\begin{array}{c}\text { Density } \\
\rho, \mathrm{kg} / \mathrm{m}^{3}\end{array}$ & $\begin{array}{c}\text { Specific heat } \\
c_{p}, \mathrm{~J} / \mathrm{kg} \cdot \mathrm{K}\end{array}$ & $\begin{array}{c}\text { Thermal conductivity } \\
k, \mathrm{~W} / \mathrm{m} \cdot \mathrm{K}\end{array}$ \\
\hline Corrugated cardboard & 4.1 & 145 & 1338 & 0.064 \\
\hline Heating tray (HDPE) & 2.3 & 950 & 1900 & 0.5 \\
\hline Heater $(\mathrm{Mg} / \mathrm{saline})$ & 6.3 & 1000 & 3284 & 171 \\
\hline Food tray $(\mathrm{PP} / . .)$. & 0.8 & 900 & 1800 & 0.2 \\
\hline Food $($ water/...) & 40 & 1000 & 4180 & 0.6 \\
\hline Lid $(\mathrm{Al} /$ olefin/...) & 0.2 & 2000 & 896 & 204 \\
\hline Air & 3.4 & 1.16 & 1005 & 0.026 \\
\hline
\end{tabular}




\section{Mathematical model}

The governing equations for heat conduction through the components of the four-food tray heating system shown in fig. 1 can be expressed as

Cardboard: $\quad \rho_{c} c_{p, c} \frac{\partial T}{\partial t}=\nabla\left(k_{c} \nabla T\right)$

Heating tray: $\quad \rho_{p e} c_{p, p e} \frac{\partial T}{\partial t}=\nabla\left(k_{p e} \nabla T\right)$

Heater:

$$
\rho_{h} c_{p, h} \frac{\partial T}{\partial t}=\nabla\left(k_{h} \nabla T\right)+q
$$

Food tray:

$$
\rho_{p p} c_{p, p p} \frac{\partial T}{\partial t}=\nabla\left(k_{p p} \nabla T\right)
$$

Food:

$$
\rho_{f} c_{p, f} \frac{\partial T}{\partial t}=\nabla\left(k_{f} \nabla T\right)
$$

Lid:

$$
\rho_{l} c_{p, l} \frac{\partial T}{\partial t}=\nabla\left(k_{l} \nabla T\right)
$$

Air:

$$
\rho_{a} c_{p, a} \frac{\partial T}{\partial t}=\nabla\left(k_{a} \nabla T\right)
$$

The boundary conditions can be written as

On bottom:

$$
\mathbf{n} \cdot\left(k_{c} \nabla T\right)=0
$$

On top:

$$
-\mathbf{n} \cdot\left(k_{c} \nabla T\right)=h\left(T-T_{a m b}\right)
$$

The initial condition can be written as

$$
\text { At } t=0 \quad T=T_{\text {init }}
$$

The source term $q$ in eqn (3) represents the heat generation rate per unit volume generated by the heater once activated. The requirements on magnesiumiron heater module currently used in the UGR-E are listed in the specification sheet MIL-DTL-32235/1 [11]. Details on the ingredients and operation are described in the U.S. Patent 5,611,329 [6]. The heat generation is based on the exothermic reaction of reactive magnesium and water, which can be expressed as 


$$
\mathrm{Mg}+2 \mathrm{H}_{2} \mathrm{O} \rightarrow \mathrm{Mg}(\mathrm{OH})_{2}+\mathrm{H}_{2}+\{\text { Heat }\}
$$

The military specifications MIL-R-44398 [10] and MIL-DTL-32235/1 [11] describe the performance/capacity test for single heater for small MRE heater and large UGR-E heater, respectively. In the performance test for a heater, a food pouch or polymeric food tray filled with water is put on top of the heater in a heating tray. After the activation water is added into the heating tray, it is covered by a lid. Temperature rise is then recorded. In order to model the heat generation from a heater, a thermodynamic approach is employed for the control volume including the heater and the test food pouch, bounded by the heating tray and the lid in the heater capacity test. For simplicity, it is assumed that there is no heat transfer through the boundary of the control volume. The governing equations of reaction and heat conduction for the system can be written as

$$
\begin{gathered}
\frac{d}{d t} m_{r}=-\lambda m_{r} \\
Q=\lambda m_{r} \Delta H_{r x n} \\
\left(m_{h} c_{p, h}+m_{w} c_{p, w}\right) \frac{d T}{d t}=Q
\end{gathered}
$$

By solving eqns (11) to (13) with some proper simplifications, an approximate analytical solution for the temperature rise and heat generation rate can be found to have the forms

$$
\begin{gathered}
\Delta T=\Delta T_{0}[1-\exp (-\lambda t)] \\
Q=Q_{0} \exp (-\lambda t)
\end{gathered}
$$

The total heat generation can be found as

$$
G=\int_{0}^{\infty} Q d t=\frac{Q_{0}}{\lambda}
$$

Assuming that the heat source is uniformly distributed over the entire space for the heater, the heat generation rate per unit volume can be found as

$$
q=\frac{Q_{0}}{V_{h}} \exp (-\lambda t)
$$

\section{Numerical solution}

The ambient and initial temperatures are taken as $T_{a m b}=23^{\circ} \mathrm{C}, T_{\text {init }}=4^{\circ} \mathrm{C}$, which are consistent with testing conditions given in [11]. The dimensions in $x$ - 
direction (thicknesses of layers in tray the stack) and values of the physical properties of the materials in the system are listed in table 1 . The heat transfer coefficient on top of the domain is assigned a typical value for natural convection as $h=10 \mathrm{~W} / \mathrm{m}^{2} \mathrm{~K}$

Lamensdorf [6] described a heater for use with an 8-ounce (226.8 g) MRE packet. The heater contains a mixture of $7.5 \mathrm{~g}$ magnesium 5 atomic weight percent iron supercorroding alloy and $1.5 \mathrm{~g}$ of nonreactive agents. The experimental data from the capacity test reported in [6] were reproduced as shown in fig. 2. The curve fitting using the form in eqn (14) yields

$$
\Delta T=71.5[1-\exp (-0.00295 t)]
$$

Then the heat generation rate from the MRE heater can be found as

$$
Q=229 \exp (-0.00295 t)
$$

Using eqn (19), the heat generation of the heater can be calculated from eqn (16) as $77.6 \mathrm{~kJ}$. The results then can be extended to the larger heater used for the group meals heating unit UGR-E based on the fact that heat generation is proportional to the mass of the reactive agent. A heater that contains $60 \mathrm{~g}$ of reactive magnesium ( 8 times the compared to that of the MRE heater) is considered. The heat generation of this heater can be calculated as $620.8 \mathrm{~kJ}$. From eqns (15) to (17), the heat generation rate per unit volume of each UGR-E heater can be found as

$$
q=4 \times 10^{6} \exp (-0.00295 t)
$$

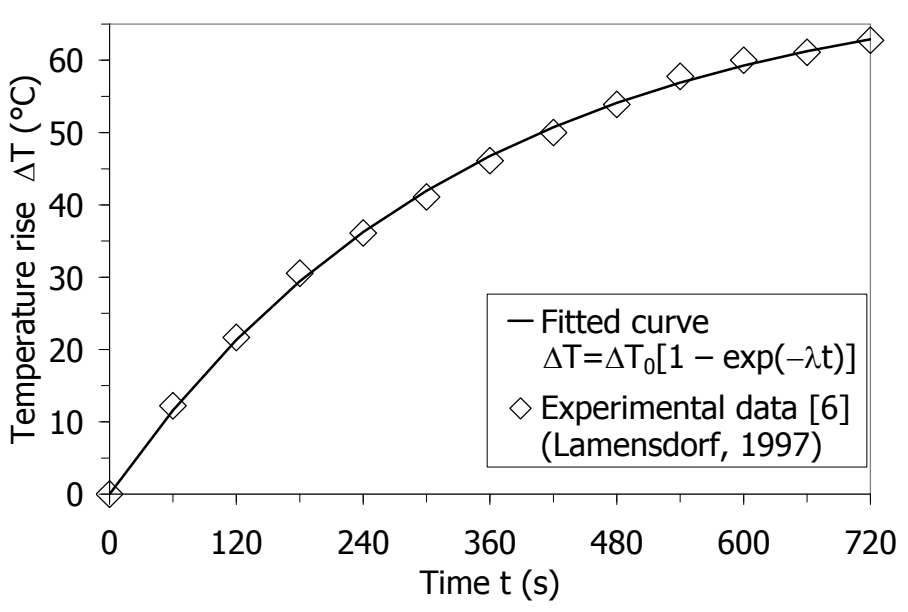

Figure 2: $\quad$ Curve fitting of experimental data from [6]. 


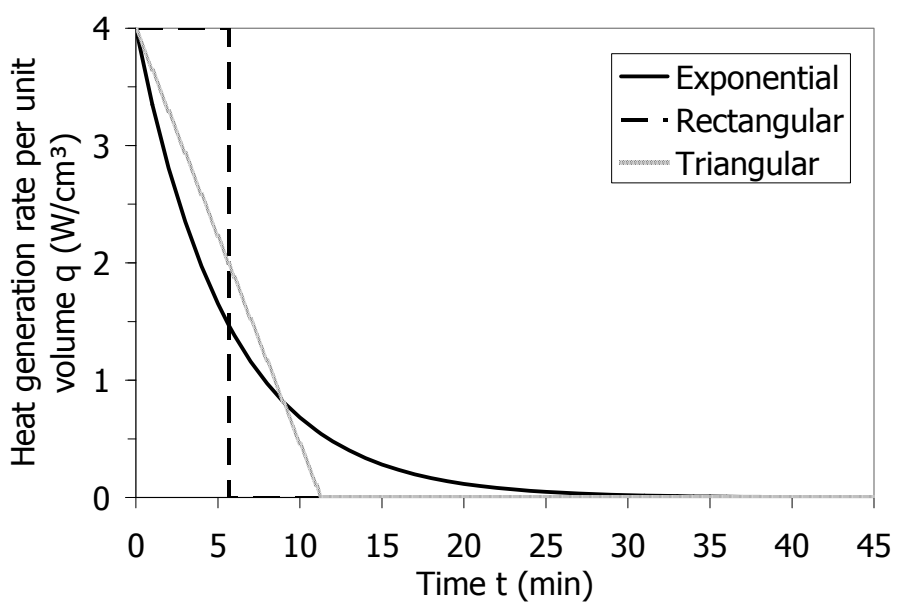

Figure 3: Heating profiles under study.

Equation (20) can be plotted as shown in fig. 3. From eqn (16), the heat generation $G$ is obviously proportional to the area under this curve. Rectangular and triangular heating profiles are also considered as shown in fig. 3. These heating profiles have the same initial heat generation rate and produce the same heat as that of the exponential heating profile.

To solve the heat conduction problem using the finite element method, the computational domain needs to be discretized into a grid or mesh of small elements. For better accuracy, the mesh needs to have finer size elements (or higher mesh density) next to the boundaries where there are high temperature gradients. The temperature in each element was approximated by using the finite element method, which led to a set of nonlinear algebraic equations that defined the discretized continuum. The software COMSOL [13] was used to solve for the numerical solution. The system of heat conduction equations was solved using the time-dependent solver. The convergence criteria of relative and absolute errors of the solutions were used with tolerances set at 0.001 and $0.000001^{\circ} \mathrm{C}$, respectively.

\section{Results and discussion}

Figure 4 presents the results for the base case where all four heaters are identical and capable of generating heat of $620.8 \mathrm{~kJ}$ with an exponential heating profile and a decay factor of $\lambda=0.295 \mathrm{~s}^{-1}$ as shown in fig. 3. Figure 4 shows the distribution of temperature over the entire computational domain at selected times of 1, 10, 20, 30, and 45 minutes. It can be observed that temperature distribution within each heater is almost uniform whereas it changes significantly within the food body of the four trays. The temperature profile at early times $(t=$ $1 \mathrm{~min}, 10 \mathrm{~min}$, etc.) shows four peaks which are corresponding to the locations of the heaters. At $t=45 \mathrm{~min}$, the temperature profile is smoothed out with a high temperature at the bottom and low temperature at the top of the stack of trays. 


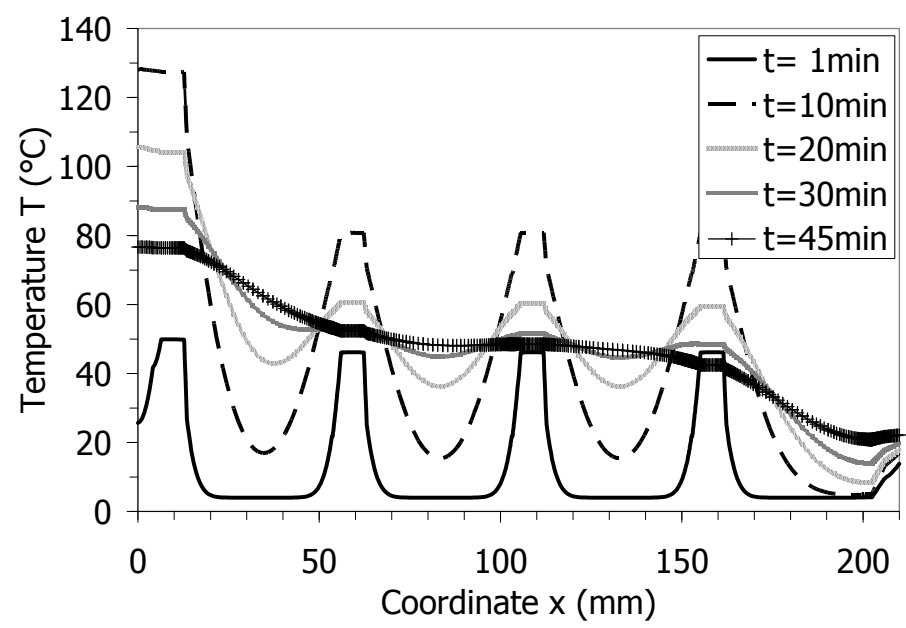

Figure 4: Temperature distribution at selected times.

The solutions corresponding to rectangular and triangular heating profiles give the similar temperature profiles as that of exponential one as shown in fig. 4. At $t=45 \mathrm{~min}$, the resulting temperature profiles corresponding to the three heating profiles are nearly identical. The differences between these thermal responses are represented by the temperature at the centres of the four food trays (denoted as F1, F2, F3, and F4 corresponding to tray order from top to bottom in the tray stack) as functions of time as shown in fig. 5. In fig. 5a, centre temperatures in the bottom and top trays are presented. It can be observed that the rectangular, triangular, and exponential heating profiles result in higher, medium, and lower temperature, respectively, in that order, most significantly in the interval of $t=10-20 \mathrm{~min}$. At $t=45 \mathrm{~min}$, centre temperature are almost the same in each food tray, despite the heating profiles. In the bottom and top trays, centre temperature reaches $63-64^{\circ} \mathrm{C}$ and $28-29^{\circ} \mathrm{C}$, respectively, for all heating profiles.

The same response can be observed in fig. $5 \mathrm{~b}$ for the two intermediate trays, 2 and 3, where the rectangular, triangular, and exponential heating profile gives the higher, medium, and lower centre temperature. Furthermore, for each heating profile, centre temperature in tray 2 and 3 are almost identical. This is because both tray 2 and 3 have the same heating configuration within the tray stack. At $t$ $=45 \mathrm{~min}$, centre temperature in tray 2 is $48-49^{\circ} \mathrm{C}$ while it is $47^{\circ} \mathrm{C}$ in tray 3 for all heating profiles, with only about $1-2^{\circ} \mathrm{C}$ difference.

From fig. 4 and similar results for other heating profiles, as well as fig. 5, it can be recognized that if all four heaters in the system are identical, temperature distribution at $t=45$ min will always be different from food tray to food tray, despite the heating profile of the heater. One significant difference is that in food tray 1 (bottom tray) temperature is much higher and less uniform compared to that of tray 2 and 3. It is caused by the use of one heater at the bottom of the stack to supply heat to only one food tray 1, whereas each other heater has to supply heat to two food trays on both its sides. This suggests that the change in 
heat generation of the bottom heater can improve the thermal behaviour of the system.

Different values of heat generation in the bottom heater were tried to find the optimum one. It is found that at $G_{1}=0.55 G_{b}$, a better temperature profile can be achieved. Figure 6 shows the temperature distribution with the heat generation in the bottom heater being changed to $G_{1}=0.55 G_{b}=341.4 \mathrm{~kJ}$ while the other heaters remain at their base heat generation value. It is observed that with reasonable temperature uniformity is achieved with temperature variation within tray 1 of about $2^{\circ} \mathrm{C}$ for all heating profiles. This observation has a significant meaning since it provides a way of saving energy material and weight of heater, while maintaining adequate heating power and improving temperature uniformity in the multi-tray food-heating unit.

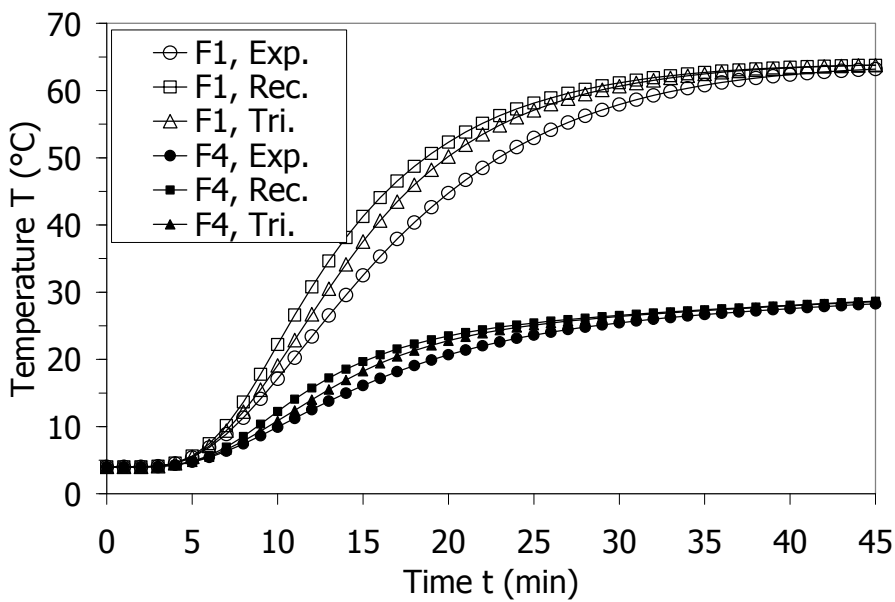

(a)

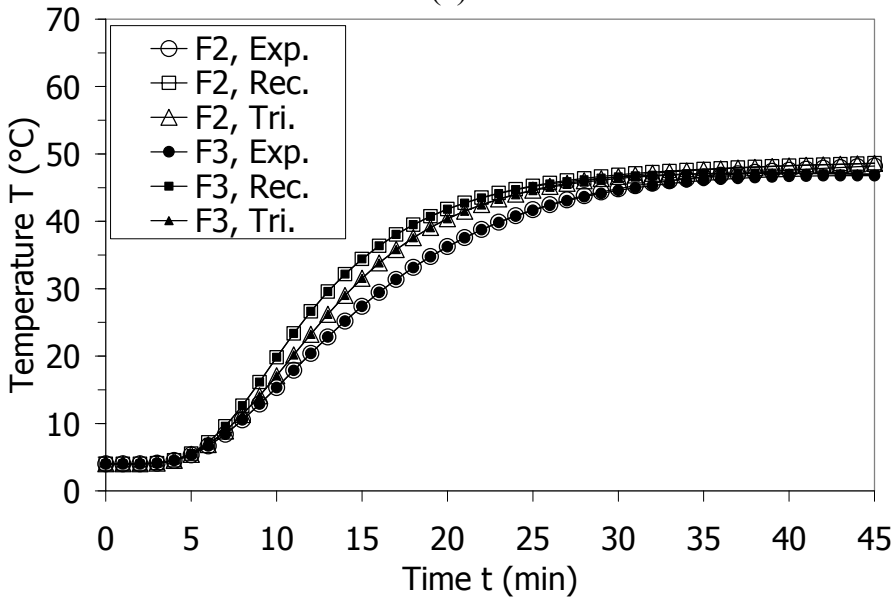

(b)

Figure 5: Temperature at centres of food trays as functions of time. 


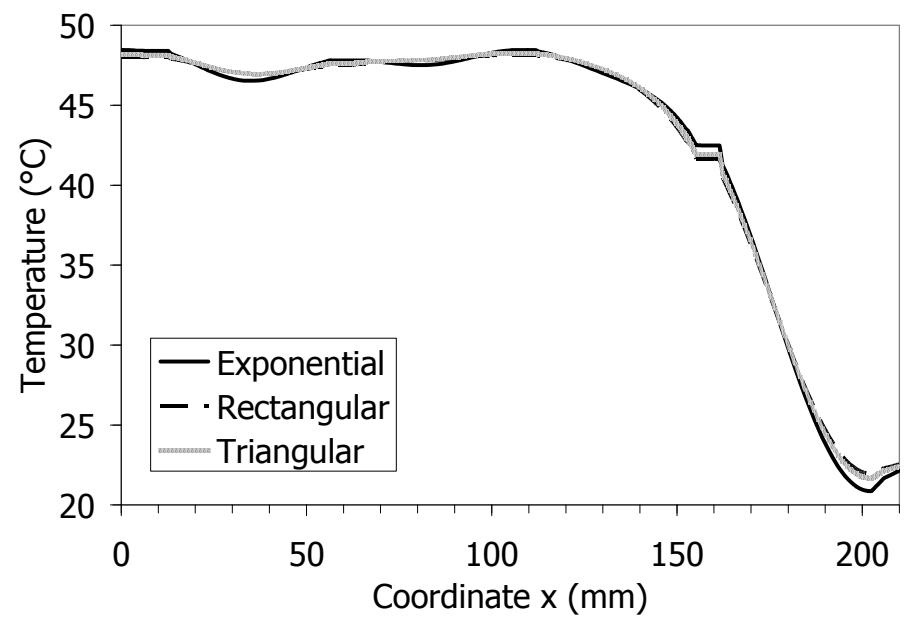

Figure 6: Temperature distribution with $G_{1}=0.55 G_{b}$.

\section{Conclusions}

The numerical simulations provide insight to heat transfer in reactive and nonreactive systems and are instrumental for design of the food self-heating system. Exponential decay, rectangular, and triangular heating profiles were considered. Centre temperature in a food tray gradually increases from initial temperature to an end temperature at 45 minutes after activation. The results show that the heating profile can affect the thermal response of the system earlier in the operating time. However, at 45 minutes after activation of the heaters, the centre temperature of food in each tray is almost the same for all heating profiles with a difference of about $1^{\circ} \mathrm{C}$. This suggests that given enough heating time, the heating profile has no significant effects on the final temperature distribution. The heat generation from each heater is the parameter that can give effective control over the thermal response of the food heating system.

It is expected from a food-heating unit that after a heating period, e.g. 45 minutes, (i) the food temperature is above a desired temperature, and (ii) the food temperature is uniformly distributed within the food body. The first criterion can be satisfied by utilizing heaters of high enough heat generation. The use of heater of lower heat generation for the bottom tray can improve the temperature difference between the bottom tray 1 and the middle trays 2 and 3 despite the heating profile from the chemical reaction. A combination of heaters with heat generation of $0.55,1,1$, and 1 times of $G_{b}$, respectively for trays $1,2,3$, and 4 in the system under study yields a food temperature of $45-50^{\circ} \mathrm{C}$ in trays $1-3$. Since food temperature in the top tray 4 is dependent on the ambient temperature on its top and on one heater on its bottom, it is difficult to control the temperature uniformity there. 


\section{Nomenclature}

$c_{p} \quad$ specific heat, $\mathrm{J} / \mathrm{kg} \mathrm{K}$

$k$ thermal conductivity, $\mathrm{W} / \mathrm{m} \mathrm{K}$

$G$ heat generation, J

$h$ heat transfer coefficient, $\mathrm{W} / \mathrm{m}^{2} \mathrm{~K}$

$k$ thermal conductivity, $\mathrm{W} / \mathrm{m} \mathrm{K}$

$m$ mass, $\mathrm{kg}$

$Q \quad$ heat generation rate, $\mathrm{W}$

$q$ heat generation rate per unit volume, $\mathrm{W} / \mathrm{m}^{3}$

$T$ temperature, ${ }^{\circ} \mathrm{C}$

$V \quad$ volume, $\mathrm{m}^{3}$

\section{Greek symbols}

$\Delta H_{r x n}$ enthalpy change of reaction, $\mathrm{J} / \mathrm{kg}$

$\Delta T$ temperature rise, ${ }^{\circ} \mathrm{C}$

$\delta \quad$ thickness, $\mathrm{m}$

$\lambda$ reaction rate constant, $\mathrm{s}^{-1}$

$\rho$ density, $\mathrm{kg} / \mathrm{m}^{3}$

\section{Subscripts}

$\begin{array}{ll}0 & \text { maximum } \\ a & \text { air } \\ a m b & \text { ambient } \\ b & \text { base case } \\ c & \text { cardboard } \\ f & \text { food } \\ h & \text { heater } \\ \text { init } & \text { initial } \\ l & \text { lid } \\ p e & \text { polyethylene (heating tray material) } \\ p p & \text { polypropylene (food tray material) } \\ r & \text { reactive magnesium } \\ w & \text { water (in test pouch) }\end{array}$

\section{Acknowledgements}

The authors would like to thank TempTroll, LLC, Tampa, Florida, and Florida High Tech Corridor Matching Grant Program for financially supporting this research project.

\section{References}

[1] Caldwell, W.A. \& Gillies, J., Development of self-heating food can. Industrial Chemist, 26 (306), pp. 301-304, 1950. 
[2] Anon, Progress of self-heating can in UK. Tin, pp. 141-142, 1960.

[3] Oliver-Hoyo, M.T., Pinto, G. \& Llorens-Molina, J.A., The chemistry of self-heating food products - an activity for classroom engagement. $J$. Chem. Educ., 86 (11), pp. 1277-1280, 2009.

[4] Kolb, K.W., U.S. Patent 7,004,161: Insertable thermotic module for selfheating cans. United States Patent and Trademark Office, 2006.

[5] Pickard, D.W., Trottier, R.L. \& Lavigne, P.G., U.S. Patent 5,355,869: Selfheating group meal assembly and method of using same. United States Patent and Trademark Office, 1994.

[6] Lamensdorf, M., U.S. Patent 5,611,329: Flameless heater and method of making same. United States Patent and Trademark Office, 1997.

[7] MIL-DTL-32235: Military Specification for Heater Module. Defense Logistics Agency (DLA), Defense Supply Center Philadelphia (DSCP), 2007. www.dscp.dla.mil/subs/support/specs/mil/32235.asp

[8] Cox, M., Feed hot food to 18 soldiers fast. Army News, Oct. 30, 2006. www.armytimes.com/legacy/new/1-292925-2320362.php

[9] What's hot at Natick Soldier RD\&E Center - Combat feeding: Coming to a theater near you, Natick Soldier Research, Development and Engineering Center (NSRDEC), 2008. www.natick.army.mil/soldier/hot/content.htm

[10] MIL-R-44398B: Military Specification: Ration Supplement, Flameless Heater, for Meal, Ready-to-Eat, Defense Logistics Agency (DLA), Defense $\begin{array}{llll}\text { Supply Center } & \text { Philadelphia } & \end{array}$ www.dscp.dla.mil/subs/support/specs/mil/44398.asp

[11] MIL-DTL-32235/1: Detail Specification Sheet - Heater Module, Type I: Magnesium and Iron Heater, Defense Logistics Agency (DLA), Defense $\begin{array}{llll}\text { Supply Center } & \text { Philadelphia }\end{array}$ www.dscp.dla.mil/subs/support/specs/mil/32235.asp

[12] UGR-E-001: Unitized Group Ration Express, Defense Logistics Agency (DLA), Defense Supply Center Philadelphia (DSCP), 2007. www.dscp.dla.mil/subs/support/specs/pcrs/ugr/ugre.asp

[13] COMSOL AB, COMSOL Multiphysics software, version 3.4, www.comsol.com 